\title{
Um Panorama sobre a Acessibilidade para Transparência em Sites de Institutos Federais do Centro-Oeste do Brasil
}

\author{
Camila L. Silva ${ }^{1}$, João Augusto Felberg ${ }^{1}$, João Cabano ${ }^{1}$, Anderson C. Lima ${ }^{1}$ \\ ${ }^{1}$ Campus de Ponta Porã - Universidade Federal de Mato Grosso do Sul (UFMS) \\ Rua Itibiré Vieira, Km 4.5, s/n - 79907-414 - Ponta Porã - MS - Brasil \\ \{caahmilk, joao.felberg, joao.cabano, anderson.correa.lima\}@gmail.com
}

\begin{abstract}
The objective of this study is to verify the degree of accessibility on Federal Institutes (FI) websites of the central-western region of Brazil. FI's are public institutions of excellence in technical and higher education in the country. Each year, thousands of candidates and students seek information and services on the FI's websites. This is a vast public and possibly also consists of people with some degree of disability. To make sure that the websites of educational institutions are accessible is an indispensable task, and, if its case, is possible to suggest the insertion of accessibility recommendations, which are prerequisites for transparent and universal access to information
\end{abstract}

Resumo. Este trabalho tem por objetivo verificar o grau de acessibilidade em websites dos Institutos Federais (IF's) da região centro-oeste do Brasil. Os IF's são instituições públicas de excelência no ensino técnico e superior no país. A cada ano milhares de candidatos e alunos buscam informações e serviços nos websites dos IF's. Este é um público vasto e que possivelmente também é composto por pessoas com algum grau de deficiência. Verificar se os websites de instituições de ensino são acessíveis é uma tarefa imprescindivel, pois se for o caso, é possível sugerir a inserção de recomendações de acessibilidade, que são pré-requisitos para um acesso transparente e universal da informação.

\section{Introdução}

De acordo com a Lei de acesso à informação pública, Lei $\mathrm{n}^{\circ} 12.527 / 2011$, qualquer pessoa pode ter acesso a informações que estejam sob a guarda de órgãos públicos, em todos os poderes. Assim sendo, garantir a acessibilidade em sítios governamentais é uma tarefa de extrema importância, pois por meio dela todo indivíduo, independente de suas condições físicas ou mentais, pode ter um acesso mais facilitado às informações prestadas nesses sítios. Ao seguir as normas de acessibilidade, os websites tornam o processo de acesso a informação mais facilitado e transparente para todo e qualquer cidadão.

A principal motivação deste trabalho decorre do fato de que apesar da acessibilidade em websites ser uma questão do novo século, são poucos os trabalhos que abordam a verificação deste quesito em sítios de instituições públicas de ensino, particularmente em Institutos Federais (IF's). Em contrapartida, o Governo Federal Brasileiro já promove a adoção do Modelo de Acessibilidade em Governo Eletrônico (eMAG) [eMAG Governo Federal 2005] nos websites das instituições públicas. O eMAG tem o

Trabalho elaborado com o apoio do Programa de Educação Tutorial (PET/SESu/MEC). 
compromisso de ser o norteador no desenvolvimento e na adaptação de conteúdos digitais do governo federal, garantindo o acesso a todos. As recomendações do eMAG permitem que a implementação da acessibilidade digital seja conduzida de forma padronizada, com fácil implementação, coerente com as necessidades brasileiras e em conformidade com os padrões internacionais. É importante ressaltar que o eMAG trata de uma versão especializada do documento internacional WCAG (Web Content Accessibility Guidelines: Recomendações de Acessibilidade para Conteúdo Web) [W3C Initiative 2008] voltado para o governo brasileiro, porém o eMAG não exclui qualquer boa prática de acessibilidade do WCAG. O WCAG é o principal documento internacional para verificação de acessibilidade em websites da internet.

Alguns trabalhos correlatos [Pereira et al. 2013, Mota et al. 2014, de Almeida et al. 2016, DE ABREU et al. 2014], tiveram por objetivo identificar quais eram as dificuldades relacionadas às normas de acessibilidade em websites de instituições públicas brasileiras. A partir destes trabalhos foi possível determinar o grau de acessibilidade de diversos sítios públicos eletrônicos. Estes trabalhos foram realizados por meio de recomendações dos modelos WCAG e eMAG, utilizando para tal as ferramentas verificadoras de acessibilidade: Ases [ASES - Governo Federal 2015] e W3C [W3C 1997]. Ases e W3C são ferramentas disponibilizadas online, que realizam por meio de métricas, uma avaliação sobre o nível de acessibilidade em um sítio eletrônico.

Em se tratando da verificação de acessibilidade de instituições públicas de ensino brasileiras, constatou-se que apesar da importância como centros de ensino inclusivos, não são encontrados trabalhos, que realizassem os testes de acessibilidade nos websites dos IF's por regiões brasileiras. É objetivo deste trabalho realizar um levantamento inicial sobre a questão de acessibilidade em websites dos Institutos Federais Brasileiros por região. Nesta primeira etapa, por uma questão de localização geográfica da pesquisa, foi escolhida a região Centro-Oeste do país. O levantamento foi realizado utilizando o verificador de acessibilidade DaSilva [Acessibilidade Brasil 2006]. A partir das informações coletadas foi realizada uma análise dos principais erros encontrados pela ferramenta, que aqui são acompanhados de recomendações para correção de cada erro.

\section{Referencial Teórico}

Nesta seção é apresentado o referencial teórico necessário para a fundamentação deste trabalho. São discutidas seções sobre: a importância da acessibilidade na web, os principais modelos de verificação, a transparência de informação e por fim a metodologia aplicada no projeto.

\subsection{Acessibilidade e Tecnologia}

Segundo censo realizado pelo IBGE, no ano de 2010 estimava-se que existiam 45 milhões de pessoas com algum tipo de deficiência no Brasil, isto equivale a 23,92\% do número total de habitantes do país [Ferreira 2016]. Deste percentual, 1,7\% estão localizados na região Centro-Oeste do país, o que totaliza 1,4 milhões de pessoas que apresentam algum tipo de deficiência na região. A distribuição proporcional de pessoas com algum tipo de deficiência no Brasil é representada pela Figura 1.

Muitas vezes a deficiência não é severa o suficiente a ponto de tornar-se uma barreira à utilização do computador. Entretanto, na maioria das páginas da web, pessoas 
cegas ou com baixa visão, pessoas com deficiência auditiva ou com dificuldade em utilizar o mouse, por exemplo, encontram barreiras de acessibilidade, que dificultam ou impossibilitam o acesso aos seus conteúdos [eMAG Governo Federal 2005]. Muitas pessoas também apresentam outras limitações relacionadas à memória, resolução de problemas, atenção, compreensão verbal, leitura e linguística, compreensão matemática e compreensão visual. Uma pessoa com dislexia, por exemplo, pode apresentar dificuldade de leitura de uma página devido a um desenho inadequado. Por isso, um sítio desenvolvido considerando a acessibilidade deve englobar os diferentes níveis de escolaridade, de faixa etária e a possibilidade de pouca experiência na utilização do computador, além de ser compatível com as diversas tecnologias utilizadas para acessar uma página da internet[eMAG Governo Federal 2005].

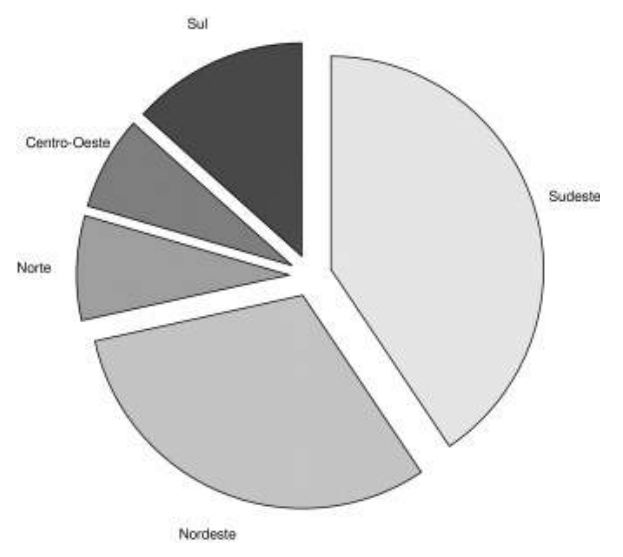

Figura 1. Representação da distribuição de pessoas com algum tipo de deficiência por regiões do Brasil.

Na sociedade atual é uma questão bastante pertinente a definição de meios, pelos quais a parcela da população com algum tipo de deficiência possa exercer da melhor forma possível, as mesmas atividades rotineiras e diárias de qualquer pessoa. Dentre estas atividades, o acesso à informação utilizando algum tipo de dispositivo tecnológico é essencial, visto que estes possibilitam a ampliação do conhecimento, a comunicação e o desenvolvimento de habilidades. O acesso a tecnologias, segundo [Castells 1999], é extremamente impactante nesta geração, logo é preciso garantir que toda a população tenha uma experiência similar, durante o processo de interação tecnológica de busca de informações. Assim sendo, o decreto $\mathrm{n}^{\mathrm{o}}$ 5.296, de 2 de dezembro de 2004, fundamentou que todo sítio e portal eletrônico de posse da administração pública deva ser desenvolvido de forma que possa transmitir suas informações e serviços a todo tipo de público usuário, garantindo assim a acessibilidade e com isto uma transparência facilitada de seus dados [DE ABREU et al. 2014].

\subsection{Modelos de Acessibilidade}

Modelos de acessibilidade abrangem um vasto conjunto de recomendações que têm como objetivo tornar o conteúdo da internet mais acessível. As recomendações buscam uniformizar o desenvolvimento dos códigos web, para assim facilitar o uso de ferramentas de acessibilidade como leitores de tela e monitores adaptados.

O cumprimento destas recomendações torna o conteúdo da internet mais acessível e transparente a um maior número de pessoas com incapacidades, incluindo cegueira e 
baixa visão, surdez e baixa audição, dificuldades de aprendizagem, limitações cognitivas, limitações de movimentos, incapacidade de fala, fotossensibilidade, bem como as que tenham uma combinação destas limitações. Seguir estas recomendações fará também com que o conteúdo web se torne mais usável aos utilizadores em geral [W3C 1997].

Dentre os modelos de acessibilidade existentes, destacam-se dois que também foram utilizados neste trabalho, são eles:

O WCAG: As Diretrizes de Acessibilidade para Conteúdo Web, em inglês Web Content Accessibility Guidelines (WCAG), constituem o modelo de recomendação para websites mais aceito no mundo, porém, cada país tem a liberdade para determinar seu próprio conjunto de diretrizes de acessibilidade [MAIA 2017]. O WCAG possui recomendações e instruções que descrevem como deve ser a estrutura de desenvolvimento de um website acessível, servindo assim de referência base para diversos outros modelos de avaliação de acessibilidade de sítios eletrônicos.

O eMAG: O Modelo de Acessibilidade em Governo Eletrônico (eMAG) consiste em uma cartilha elaborada com recomendações e boas-práticas, que devem ser implementadas em websites governamentais brasileiros, para garantir o teor de acessibilidade e uma maior transparência das informações e serviços neles contidos. Desenvolvido em 2004, as diretrizes do eMAG podem ser utilizadas para mensurar os índices de acessibilidade dos websites da esfera federal do governo brasileiro [MAIA 2017].

\subsection{O Avaliador DaSilva}

Dentre os avaliadores de acessibilidade em websites, os que foram utilizados para este trabalho são os encontrados em análises bibliográficas cujos resultados são considerados mais concisos e bem elaborados, com destaque para o avaliador Dasilva. Esta plataforma Java consiste no primeiro avaliador em português de websites, disponibilizado em www.dasilva.org.br. Utiliza como base para suas verificações os modelos de acessibilidade e-Mag e WCAG. O DaSilva avalia toda a estrutura de construção de um website. Os resultados de seus testes são gerados em ordem de prioridades dos erros (do mais relevante ao menos relevante). Ele consiste em uma aplicação cuja execução é reconhecida e utilizada pela literatura [de Sousa et al. 2008] [Santos 2016] [DE ABREU et al. 2014] [de Almeida et al. 2016] [MAIA 2017].

\subsection{Acessibilidade para Transparência}

Segundo [Araújo et al. 2010], a acessibilidade constitui um dos pilares essenciais para que o propósito de transparência de informações na internet seja facilitado. Isso porque a acessibilidade busca tornar mais igualitário o processo de obtenção de uma informação na web por pessoas portadoras ou não de algum tipo de deficiência. Se pessoas com alguma deficiência não conseguem acessar a informação ou possuem muita dificuldade neste processo, então o website em questão dificulta a transparência de seu conteúdo.

\subsection{Trabalhos Correlatos}

A acessibilidade na web, ou rede mundial de computadores, diz respeito a viabilizar que qualquer pessoa, usando qualquer tecnologia adequada à navegação web esteja apta a visitar qualquer website, obtenha a informação oferecida e interaja com o mesmo. Para que 
isso seja possível, é necessário que os criadores de páginas e sistemas web, assim como seus mantenedores, estejam atentos às recomendações de acessibilidade [Melo 2009]. Nesta seção são apresentados alguns trabalhos relacionados com este tema e que serviram de inspiração para este projeto, são eles:

- O trabalho de [Pereira et al. 2013] realizou uma análise sobre o grau de acessibilidade em sítios eletrônicos de instituições de ensino de nível superior do país. Os resultados obtidos foram utilizados em conclusões acerca dos erros de acessibilidade mais frequentes nos websites das instituições. Mostrou-se que as instituições analisadas muitas vezes não estavam em conformidade com o modelo eMAG, que foi o utilizado no trabalho.

- O trabalho de [Mota et al. 2014] efetuou uma análise sobre o grau de acessibilidade nos portais eletrônicos dos institutos federais localizados no estado de Minas Gerais. Foi realizada uma medição da acessibilidade por meio das ferramentas online ASES (Avaliador e Simulador de Acessibilidade de Sítios) e daSilva.org.br [ASES - Governo Federal 2015, Acessibilidade Brasil 2006]. Neste caso, verificou-se que os websites apurados continham inconformidades com o eMAG. Como conclusão principal, constatou-se que um dos principais motivos era o número reduzido de funcionários de tecnologia da informação especializados sobre o assunto dentro das instituições.

- O trabalho [de Almeida et al. 2016] buscou propor recomendações acerca da inserção da acessibilidade em websites. O trabalho utilizou o método automático de avaliação de acessibilidade TAW. O TAW é uma ferramenta do W3C (World Wide Web Consortium) e foi utilizado para verificar os websites de diversas instituições federais de ensino superior. De acordo com os erros coletados durante a avaliação, foram fornecidas sugestões para evitá-los.

- Em seu trabalho [DE ABREU et al. 2014] realizou uma análise sobre quatorze portais de informação das prefeituras de cidades do estado de Rio de Janeiro, mais especificamente, da região Sul Fluminense do estado. Ele constatou que apenas um dos sítios analisados estava em conformidade com as diretrizes especificadas pelo eMAG. Em sua conclusão ele descreveu sobre a necessidade e a importância do vínculo entre transparência de informações e acessibilidade.

- Com enfoque na acessibilidade de analfabetos funcionais, [Capra et al. 2013] realizou um levantamento aplicado em dois websites: um do Ministério do Trabalho e Emprego (MTE) e outro do Ministério da Previdência Social (MPS). A análise utilizou a fórmula de inteligibilidade Flesch Reading Ease e a ferramenta CohMetrix-Port [Scarton and Aluísio 2010]. De acordo com os resultados obtidos concluiu-se que tanto a acessibilidade dos websites, quanto os testes de usabilidade foram insatisfatórios. Os verificadores apontaram falhas no desenvolvimento das aplicações.

\section{Metodologia e Desenvolvimento}

Para a tarefa de avaliação de acessibilidade em websites de Institutos Federais Brasileiros da região Centro-Oeste, o processo de desenvolvimento deste trabalho foi dividido em etapas, são elas:

1. Análise de trabalhos correlatos: verificação de trabalhos similares na literatura realizando uma análise de suas perspectivas e resultados. 
2. Levantamento e escolha dos websites para análise de acessibilidade: optou-se neste trabalho pelos sítios de Institutos Federais da região Centro-Oeste, devido a localização geográfica e ao conhecimento dos autores sobre a região. Particularmente, não foram encontrados trabalhos que abordem o segmento de IF's por regiões na literatura. Entretanto esta análise é urgente, dada a importância ao acesso de conteúdo de forma clara e íntegra, por qualquer estudante ou candidato a ingresso nestes centros de ensino públicos e de excelência.

3. Definição de avaliador: Escolha de um avaliador de acessibilidade para a tarefa de levantamento de erros e inconsistências dos websites. Dentre os avaliadores disponíveis escolheu-se o DaSilva, por ser uma ferramenta gratuita e abrangente, que realiza uma varredura no documento $w e b$ validando acessibilidade tanto pelas diretrizes do WCAG, quanto do eMAG.

4. Análise dos resultados obtidos: Foi realizada uma análise dos resultados, identificando as mais graves inconsistências das principais recomendações e sugerindo soluções que pudessem supri-las.

\subsection{Os Websites Selecionados}

A implantação dos Institutos Federais no Brasil é um projeto progressista que entende a educação como compromisso de transformação e de enriquecimento de conhecimentos objetivos capazes de modificar a vida social e de atribuir-lhe maior sentido e alcance no conjunto da experiência humana, proposta incompatível com uma visão conservadora de sociedade. Trata-se, portanto, de uma estratégia de ação política e de transformação social [Pacheco 2011]. Assim sendo garantir o acesso universal as informações dos websites dos IF's é também uma tarefa de transformação social. É preciso então garantir que o acesso a estas informações seja acessível e possua transparência facilitada.

\section{Resultados}

Como já descrito, para a verificação de acessibilidade utilizou-se a ferramenta de avaliação DaSilva. Foram pesquisados os sítios eletrônicos das unidades dos Institutos Federais presentes nos estados e no distrito federal da região Centro-Oeste do Brasil. Os resultados desta análise foram compilados na Tabela 1.

\begin{tabular}{|l|c|c|c|c|c|c|c|}
\cline { 3 - 8 } \multicolumn{2}{c|}{} & \multicolumn{9}{c|}{ Média de Erros } & \multicolumn{2}{c|}{ Média de Alertas } \\
\hline IF's-Regiões & Sites Avaliados & P1 & P2 & P3 & e-MAG & P1+P2+P3 & e-MAG \\
\hline IF's da Reg. 1 & 7 & 16 & 11 & 0 & 23 & 142 & 20 \\
\hline IF's da Reg. 2 & 15 & 113 & 5 & 0 & 88 & 173 & 49 \\
\hline IF's da Reg. 3 & 5 & 22 & 14 & 0 & 34 & 212 & 50 \\
\hline IF's da Reg. 4 & 10 & 23 & 4 & 0 & 45 & 386 & 34 \\
\hline
\end{tabular}

Tabela 1. Análise de acessibilidade do IF’s da região Centro-Oeste.

\subsection{Análise de Resultados}

A primeira coluna da Tabela 1 apresenta as quatro regiões pesquisadas. Em uma mesma região geralmente existem diversas unidades de IF's. A segunda coluna apresenta o número de websites avaliados por unidades de IF's de cada região pesquisada. As colunas 
três e quatro contemplam a média de erros e média de alertas encontrados na varredura de acessibilidade dos websites por meio do avaliador. A média de erros foi categorizada dispondo erros encontrados pelo WCAG e pelo eMAG. Em particular, os erros e alertas do WCAG foram divididos em prioridade grave (P1), prioridade média (P2) e prioridade não relevante $(\mathbf{P 3})$. As médias totais descritas na última linha foram calculadas pela seguinte fórmula: Média Total $=$ Total de Erros $/$ Número de Regiões, por exemplo, para erros graves (P1) a média calculada foi igual a 43,5 que é igual a 174 / 4.

\section{Média = Número de Erros Graves / Número de IF's}

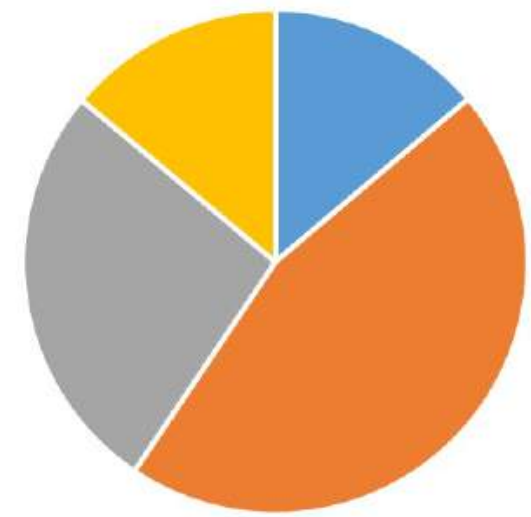

- Região 1 = 2,29 = Região $2=7,53$

“ Região $3=4,40$ " $\operatorname{Região~} 4=2,30$

Figura 2. Análise de erros graves nos IF's de cada região.

O gráfico representado pela Figura 2 apresenta a média do número de erros graves por IF's em cada região. Pode-se perceber que a região 2 apresentou a média com o maior índice de erros.

Por meio destes resultados percebe-se que a quantidade de erros graves encontrados nos websites dos IF's das quatro regiões pesquisadas é bastante acentuado. Este é um fator crítico, pois revela que existe uma grande dificuldade no processo de acesso ao conteúdo por pessoas com algum tipo de deficiência. Os erros de categoria média (P2) também possuíram um número relevante, o que corrobora ainda mais na dificuldade de acessibilidade dos websites.

\subsection{Principais Recomendações de Acessibilidade Inconsistentes e Comuns nos IF's}

As principais recomendações de acessibilidade que geraram erros e alertas e que são comuns nos websites dos IF's dos estados pesquisados são apresentadas a seguir. Elas são acompanhadas de uma definição e da solução sugerida pelos documentos de acessibilidade WCAG e/ou eMAG. Tratam-se de recomendações (diretrizes) com relevância considerada alta, visto que o não cumprimento delas impede a acessibilidade de pessoas com algum grau de deficiência. São elas:

- Conteúdo Não Textual: Diretriz 1.1.1 do eMAG e 3.6 do WCAG. Descreve que todo conteúdo não textual que é apresentado ao usuário deve ter uma alternativa 
em texto que sirva para um propósito equivalente. Possui uma relevância considerada alta, visto que o não cumprimento da recomendação impede a acessibilidade para pessoas com algum grau de deficiência visual. A diretriz descreve que todo o acesso a informação disposto como imagens, tabelas e gráficos possua uma tradução em texto. Esta ação é bastante útil para diversas aplicações de acessibilidade, como os leitores de tela, por exemplo, que se baseiam nas informações contidas nos códigos do site, para realizar a tradução do conteúdo para linguagem natural. A forma para garantir a validade da diretriz consiste em garantir a inserção de rótulos e descrições nos elementos não textuais existentes, por meio dos recursos disponibilizados pela linguagem de programação utilizada na estruturação do conteúdo.

- Informações e Relações: Diretriz 1.7 do eMAG 1.3.1 e do WCAG. Descreve que as informações, a estrutura e as relações transmitidas através da apresentação possam ser determinadas de forma programática ou estejam disponíveis no texto. A diretriz consiste em garantir que a estrutura do website seja lógica e coerente, para que, caso a forma de apresentação do conteúdo mude, no caso de transformação para linguagem de voz, por exemplo, o contexto não seja alterado e o usuário consiga receber a informação de forma correta. A forma para garantir a validade da diretriz consiste da utilização de marcadores semânticos nas estruturas do conteúdo.

- Etiquetas ou Instruções: Diretriz 3.3.2 do WCAG. Descreve que as etiquetas ou instruções do website sejam fornecidas sempre que o conteúdo deste exigir a entrada de dados por parte do usuário. Essa recomendação procura garantir que o usuário saiba que determinado elemento do website requer entrada de dados e especifica o tipo de dado esperado, juntamente com informações relevantes sobre o uso que se dará com a informação recebida. A forma para garantir a validade da diretriz descreve que existam rótulos que associem textos a formulários, na estrutura do website.

- Nome, Função, Valor: Diretriz 4.1.2 do WCAG. Descreve que todos os componentes da interface de utilizador (incluindo, mas não se limitando a: elementos de formulário, hiperligações e componentes gerados por scripts), o nome e a função possam ser determinados de forma programática. Além disto os estados, as propriedades e os valores, que podem ser parametrizados pelo utilizador, também possam ser definidos de forma programática. A notificação das alterações a estes elementos deve estar disponível para consulta pelos agentes de utilizador, incluindo as tecnologias de apoio. A forma para garantir a validade da diretriz descreve, que caso seja utilizado alguma aplicação de interface no website, deve-se implementar medidas para garantir que os aplicativos de acessibilidade recebam as notificações corretamente.

- Teclado: Diretriz 2.1.1 do WCAG e 2.2 e 2.1 do eMAG. Descreve que é preciso permitir que todas as funcionalidades do website também possam ser desenvolvidas via interação com o teclado. Ou seja, se a única forma de o usuário interagir com o site for por meio de teclados, então é preciso garantir que as funcionalidades do website consigam ser adaptadas para operar nesta situação. A forma para garantir a validade da diretriz descreve que as aplicações sejam adaptáveis para funcionarem tanto da forma original, quanto em uma opção genérica, operável pelo teclado e isto sem mudança no possível resultado final. 
- Foco: Diretriz 6.4 do eMAG. Descreve que quando um elemento de formulário receber foco, não deva ser iniciada nenhuma mudança automática na página que confunda ou desoriente o usuário. As mudanças devem ocorrer através do acionamento de um botão. Isto porque qualquer mudança de contexto inesperada no fluxo ou conteúdo do website pode frustrar um usuário que utiliza aplicações de acessibilidade, já que a mudança pode não ser percebida tão facilmente. A forma para garantir a viabilidade da diretriz descreve que mudanças de contexto devam ser aplicadas apenas quando suas ocorrência forem explícitas com interação prévia do usuário.

- Identificação: Diretriz 3.5 do eMAG. Descreve que é preciso identificar claramente o destino de cada link, informando, inclusive, se o link remete a outro sítio. Além disso, é preciso que o texto do link faça sentido mesmo quando isolado do contexto da página. Busca garantir que o usuário tenha ciência do local para onde será redirecionado caso uma determinada ação seja realizada. A forma para garantir a viabilidade da diretriz descreve que é preciso determinar rótulos e descrições em todos elementos que realizem redirecionamentos, como botões, de forma a deixar claro para onde será feito o redirecionamento.

\section{Conclusão}

A pesquisa deste trabalho destaca que os websites dos IF's da região Centro-Oeste do país não estão em plena conformidade com as diretrizes de acessibilidade reconhecidas e aceitas. Pode-se inferir que ainda há muito trabalho a ser feito, para que as diretrizes sejam cumpridas, e consequentemente, que a informação seja acessada por todo tipo de usuário. Assim sendo, pode-se concluir que a transparência das informações nos websites dos Institutos Federais da região Centro-Oeste do Brasil também não está está sendo ofertada em totalidade, já que uma parcela dos receptores dos dados pode não estar conseguindo ter acesso aos mesmos. Percebe-se que apesar da existência de documentos com recomendações de acessibilidade para internet, os desenvolvedores da estrutura do conteúdo web destas instituições podem não estar familiarizados com as normas e leis ou não estão sendo informados sobre a necessidade de realizar adaptações necessárias para garantir a acessibilidade e assim facilitar a transparência de acesso a informação nos websites.

\section{Trabalhos Futuros}

Um trabalho futuro, que é motivação de continuidade, consiste em realizar novos experimentos sobre a acessibilidade em websites dos IF'S da região Centro-Oeste. A partir destes novos experimentos a proposta é desenvolver uma aplicação web, que seja capaz de auxiliar na correção dos erros de acessibilidade que forem encontrados, de forma a apresentar aos desenvolvedores como o código deveria estar organizado para garantir o acesso de todos os usuários.

\section{Referências}

Acessibilidade Brasil (2006). Avaliador de Acessibilidade. http: / /www . dasi lva. org . br / , note $=$ Online; accessed 19 march 2017 .

Araújo, R. M., Cappelli, C., and Leite, J. (2010). A importância de um modelo de estágios para avaliar transparência. Revista TCMRJ, setembro, (45):97. 
ASES - Governo Federal (2015). Avaliador e Simulador de Acessibilidade em Sítios. http://asesweb.governoeletronico.gov.br/ases/, note = Online; accessed 19 march 2017.

Capra, E., Benjamin, K., Ferreira, S. B. L., Capelli, C., and da Silveira, D. S. (2013). O acesso dos analfabetos funcionais ao conteúdo informacional dos sites governamentais brasileiros. In Proceedings of the 12th Brazilian Symposium on Human Factors in Computing Systems, pages 132-141. Brazilian Computer Society.

Castells, M. (1999). A era da informação: economia, sociedade e cultura., volume 1. Paz e terra.

DE ABREU, J. C. A., DE AGUIAR, A. O., and DA HORA, R. D. (2014). Internet portal south fluminense region-brazil: An exploratory study. RIEM, page 122.

de Almeida, L. R., de Brito, R. R., da Silva, A. P., de Lima Farias, Á., and de Castro Neto, D. S. M. (2016). Acessibilidade virtual: Um estudo acerca da acessibilidade, usabilidade e comunicabilidade em portais institucionais federais.

de Sousa, F. F., Osorio, M. C., and de Andrade, E. L. (2008). Proposta de avaliação de acessibilidade em sites por deficientes visuais.

eMAG Governo Federal (2005). emag - modelo de acessibilidade em governo eletrônico. http://emag.governoeletronico.gov.br/, note = Online; accessed 19 march 2017.

Ferreira, F. S. (2016). Os dois mundos da inclusão do deficiente visual.

MAIA, L. S. (2017). Uma análise preliminar da acessibilidade web dos sites de serviços de divulgação pública no brasil com base no e-mag.

Melo, A. M. (2009). Acessibilidade na web. ACESSIBILIDADE, page 33.

Mota, F. A. O., Diniz, A. P. V., Minoves, C. S., and de Paiva Oliveira, S. (2014). Acessibilidade web dos sites oficiais dos institutos federais presentes no estado de minas gerais. Anais dos Simpósios de Informática do IFNMG-Campus Januária.

Pacheco, E. (2011). Os institutos federais: uma revolução na educação profissional e tecnológica. São Paulo: Moderna.

Pereira, A. S., Machado, A. M., and Carneiro, T. C. J. (2013). Avaliação da acessibilidade dos sitios eletronicos das instituições de ensino superior brasileiras. Informação \& Sociedade, 23(3).

Santos, G. C. (2016). Aspectos de acessibilidade do governo eletrônico: Avaliação de portais web com ênfase em portadores de deficiência visual. Projetos e Dissertações em Sistemas de Informação e Gestão do Conhecimento, 4(2).

Scarton, C. E. and Aluísio, S. M. (2010). Análise da inteligibilidade de textos via ferramentas de processamento de língua natual: adaptando as métricas do coh-metrix para português.

W3C (1997). Web accessibility initiative (wai). https : / /WWW . W3 . org/WAI/, note = Online; accessed 19 march 2017.

W3C Initiative (2008). Web Content Accessibility Guidelines (WCAG). https: / / www.w3.org/WAI/intro/wcag, note = Online; accessed 19 march 2017. 